\title{
Free Urinary Glycosylated Hydroxylysine as an Indicator of Altered \\ Collagen Degradation in the Mucopolysaccharidoses
}

Nina Patel ${ }^{1,2}$, Philippa Mills ${ }^{1}$, James Davison ${ }^{2}$, Maureen Cleary ${ }^{2}$, Paul Gissen ${ }^{1,2,3,4}$, Blerida Banushi $^{3}$, Ivan Doykov ${ }^{1}$, Megan Dorman ${ }^{2}$, Kevin Mills ${ }^{1}$, Wendy E. Heywood ${ }^{1,2}$

Corresponding author: Dr Wendy E. Heywood wendy.heywood@ucl.ac.uk

Word counts: Word counts for the text (excluding summary, acknowledgments, references and figure legends) $=3156$ and the summary. 275

Figures/ table: 4

\section{Summary}

Extracellular matrix (ECM) disruption is known to be an early pathological feature of the Mucopolysaccharidoses (MPS). Collagen is the main component of the ECM and its metabolism could act as a useful indicator of ECM disruption. We have measured the specific collagen breakdown products; urinary free hydroxylated (Lys-OH) and glycosylated hydroxylysines (Lys-O-Gal and Lys-O-GalGlc) in MPS patients using a tandem LC-MS/MS assay. A pilot study cohort analysis indicated that concentrations of lysine and Lys-OH were raised significantly in MPS I (Hurler) disease patients. Lys-O-GalGlc was raised in MPS II 
and MPS VI patients and demonstrated a significant difference between MPS I Hurler and an MPS I Hurler-Scheie group. Further analysis determined an age association for glycosylated hydroxylysine in control samples similar to that observed for the glycosaminoglycans. Using defined age ranges and treatment naïve patient samples we confirmed an increase in glycosylated hydroxylysines in MPS I and in adult MPS IVA. We also looked at the ratio of Lys-O-Gal to Lys-O-GalGlc, an indicator of the source of collagen degradation, and noticed a significant change in the ratio for all paediatric MPS I, II and IV patients, and a small significant increase in adult MPS IV. This indicated that the collagen degradation products were coming from a source other than bone such as cartilage or connective tissue. To see how specific the changes in glycosylated hydroxylysine were to MPS patients we also looked at levels in patients with other inherited metabolic disorders. MPS patients showed a trend towards increased glycosylated hydroxylysines and an elevated ratio compared to other metabolic disorders that included Battens disease, Fabry disease, Pyridoxine-dependent epilepsy (due to mutations in ALDH7A1), and Niemann Pick C disease.

Synopsis: Urinary glycosylated hydroxylysine composition is altered and elevated in MPS disorders indicating altered collagen homeostasis and extracellular matrix disruption.

Details of the contributions of individual authors: NP Performed laboratory and data analysis and contributed to drafting of manuscript; PM facilitated sample collection and information and reviewed manuscript; JD provided clinical information and expertise; MC provided clinical information and expertise; PG provided specific assay reagent, samples and 
clinical information and expertise; BB performed initial pilot experiment analysis; ID performed laboratory analysis; MD consented and collected patient samples; KM contributed to conception of the study, drafting of manuscript and facilitated mass spectral analysis; WH provided funding, experimental design and drafted manuscript.

Competing interest statement: Nina Patel, Philippa Mills, James Davison, Maureen Cleary, Paul Gissen, Blerida Banushi, Ivan Doykov, Megan Dorman, Kevin Mills report no specific funding sources and/or potential conflicts of interest from each author that relate to the research covered in the article.

Dr Wendy Heywood received funds from Shire pharmaceuticals and the Great Ormond Street Hospital Biomedical Research Centre.

Ethics approval: The collection of samples for this study has ethical approval (13/LO/0168; IRAS ID 95005; London-Bloomsbury Research Ethics Committee) and Health Research Authority (HRA) approval

Informed consent statement: All procedures followed were in accordance with the ethical standards of the responsible committee on human experimentation (institutional and national) and with the Helsinki Declaration of 1975, as revised in 2000. Informed consent was obtained for all patients included in the study.

Keywords: Mucopolysaccharidoses, collagen; glycosylated hydroxylysine, biomarker 


\section{Introduction}

The introduction of therapies that target the underlying enzyme deficiency in a number of the Mucopolysaccharidoses (MPS) disorders has highlighted the need for biomarkers that would aid in the evaluation of disease burden and as a means to objectively measure therapeutic response in individual patients. It is increasingly recognised that there is a need for other biomarkers in addition to glycosaminoglycans (GAGS) to monitor treatment of the extraordinarily complex pathology observed in MPS disorders.

One of the key early pathological features in MPS disorders is disruption of the extracellular matrix (ECM) which ultimately leads on to many further pathological events (Oussoren et al 2011). Collagen is the most abundant protein in the human body and the major component of the extracellular matrix. Its turnover could be a useful indicator of ECM disruption. Glycosylated hydroxylysine (Lys-O-Gal and Lys-O-GalGlc) is a unique and specific posttranslational modification of collagen (Spiro 1969). Free glycosylated hydroxylysine is a degradation product of collagen breakdown (Krane et al 1977) and is easily measurable in urine. It is thought to be an accurate measure of collagen metabolism as it is only metabolised to a minor degree (Krane et al 1977) and excreted in different ratios depending on the tissue source. Lys-O-Gal is predominantly found in bone tissue and Lys-O-GalGlc is mainly found in skin (Pinnell et al 1971). We have developed a tandem LC-MS/MS method to measure urinary lysine, hydroxylated lysine and the two glycosylated forms of hydroxylysine (Banushi et al 2016). Using this method we have shown that there are age-related levels of glycosylated hydroxylysine in control individuals and were able to look at the levels of each 
modified lysine and also the ratio of the glycosylated hydroxylysines to determine changes in the source of the glycosylated hydroxylysine in a cohort of MPS I, II and VI urine samples. In order to validate these initial findings, we performed analyses on a separate cohort of treatment-naive MPS I, II and IV samples. The specificity of the altered glycosylated hydroxylysines for MPS was determined by comparison of samples from patients with a selection of other metabolic disorders.

\section{Methods}

\section{Samples}

Urine samples used in this study were provided by patients visiting the Metabolic Disease Clinic at Great Ormond Street Hospital London, UK. The collection of samples for this study has ethical (13/LO/0168; IRAS ID 95005; London-Bloomsbury Research Ethics Committee) and Health Research Authority (HRA) approval. All patients included consented to take part in the study. Samples were pseudo-anonymised and stored at $-80{ }^{\circ} \mathrm{C}$ within 24 hrs of collection. Sample information is given in Supplementary Data 1. Samples from patients prior to treatment, on a combination of treatments as well as those where treatment status was unknown, were analysed in the initial pilot study. Age-matched controls were surplus samples from routine diagnostic testing at Great Ormond Street Chemical Pathology Department. The second cohort of MPS patient samples used for confirmation of initial findings consisted of only pre-treatment samples. 


\section{FMOC-derivatization of amino acids for UPLC-MS/MS analyses}

Samples were analysed as described previously (Banushi et al 2016). The collagen-derived amino acids Lys, Lys-OH, Lys-O-GalGlc and Lys-O-Gal and internal standard ${ }^{13} \mathrm{C}_{6}{ }^{15} \mathrm{~N}-\mathrm{Lys}$ were derivatized with fluorenylmethyloxycarbonyl chloride (FMOC-Cl) in $60 \mu \mathrm{l}$ of spun urine using established protocols (Mills et al 2006).

\section{UPLC-MS/MS analysis}

Lys-O-GalGlc and Lys-O-Gal standards were a kind gift from Professor Ruggero Tenni (University of Pavia, Italy). A stable isotope-labelled lysine ${ }^{13} \mathrm{C}_{6}{ }^{15} \mathrm{~N}$-Lys (Sigma, UK) was used for quantitation of all targeted metabolites. A Waters Acquity Ultra Performance Liquid Chromatography (UPLC) coupled to a Xevo TQ-S Triple Quadrupole Mass Spectrometer (Waters Corp, UK) was operated in negative ion mode (MS-operating parameters are shown in Supplementary Table S2). The capillary voltage was maintained at $3.7 \mathrm{kV}$, with source temperature held constant at $150^{\circ} \mathrm{C}$ and nitrogen used as the nebulizing gas at a flow rate of $301 \mathrm{~h}-1$. FMOC-derivatised amino acids were separated using an ACQUITY UPLC $2.1 \times$ $50 \mathrm{~mm}$ BEH C8 1.7- $\mu \mathrm{m}$ column (Waters Corp). The 5 minute peptide elution gradient was as follows; $5 \%$ acetonitrile: $95 \%$ methanol to $67 \%$ acetonitrile: $33 \%$ methanol over 4 min and back to $5 \%$ acetonitrile:95\% methanol for 1 min for column re-equilibration. Flow rates were $0.8 \mathrm{ml} \mathrm{min}{ }^{-1}$ and the column temperature $40^{\circ} \mathrm{C}$.

\section{Data Analysis and Statistical methods}

Mass spectrometry data was analysed using MassLynx and TargetLynx (Waters Corp). All samples were analysed in duplicate. Analyte values were obtained by ratioing to the internal 
standard and the value determined from a standard curve. Values were then standardised to urinary creatinine values. Non-parametric testing was used in this study. A two-tailed MannWhitney P value $<0.05$ was considered significant. Spearman correlation was used to determine significant correlation and reference ranges determined by 5\% and 95\% percentiles.

\section{Results}

\section{Initial investigations of modified lysine in a test cohort of MPS patients}

To investigate if modified lysines are altered in the urine of MPS patients a test cohort of 34 patient urines were analysed (figure 1B). These samples were surplus to routine diagnostic testing and consisted of a spectrum of MPS disorders. Some samples were from patients prior to treatment, others from those on a combination of treatments and some from individuals where treatment status was unknown. The MPS I group was sub-divided into Hurler (MPS I H), Hurler/Scheie (MPS I- HS+S) and Scheie. There was no significant difference observed in the levels of modified lysines between the MPS II neurological and non-neurological subtypes, indicating that collagen turnover is not associated with the neurological phenotype of MPS II. Therefore these MPS II sub-types were combined as MPS II. Figure 1A shows the structure of the modified lysines that were measured by LC-MS/MS. Figure 1B shows the LC-MS/MS results for lysine and each of the 3 modified lysines for each MPS group. The severe phenotype MPS I Hurler group demonstrated a 2.95 and 2.68 fold increase in lysine and Lys-OH, respectively relative to controls. The glycosylated forms of hydroxylysine, 
however, showed a greater level of change in the MPS groups. Lys-O-Gal and Lys-O-GalGlc levels were significantly raised in both MPS IH and MPS II by approximately 5 and 3.8-4 fold, respectively. Lys-O-GalGlc was also significantly elevated in MPS VI by 3.9 fold and in MPS IH and MPS I HS+S 2 fold.

Previous studies have shown that the ratio of Lys-O-GalGlc to Lyso-O-Gal varies depending on whether the hydroxylysine is derived from skin (ratio of approx. 2) or bone (ratio of approx. 0.5)(Pinnell et al 1971). The ratios for the control paediatric group in this study were approximately 0.5 indicating that the primary source of collagen breakdown in urine originates from bone. Whilst the ratio in the MPS groups studied did not show a significant difference to that of controls a trend towards higher ratios was observed in both the MPS IH and MPS VI groups. 


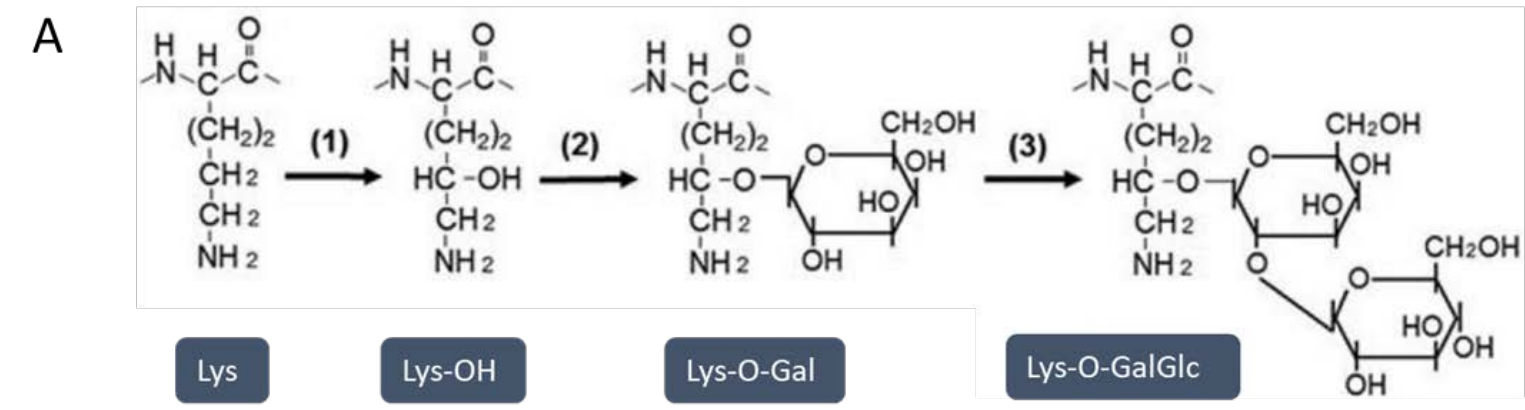

B
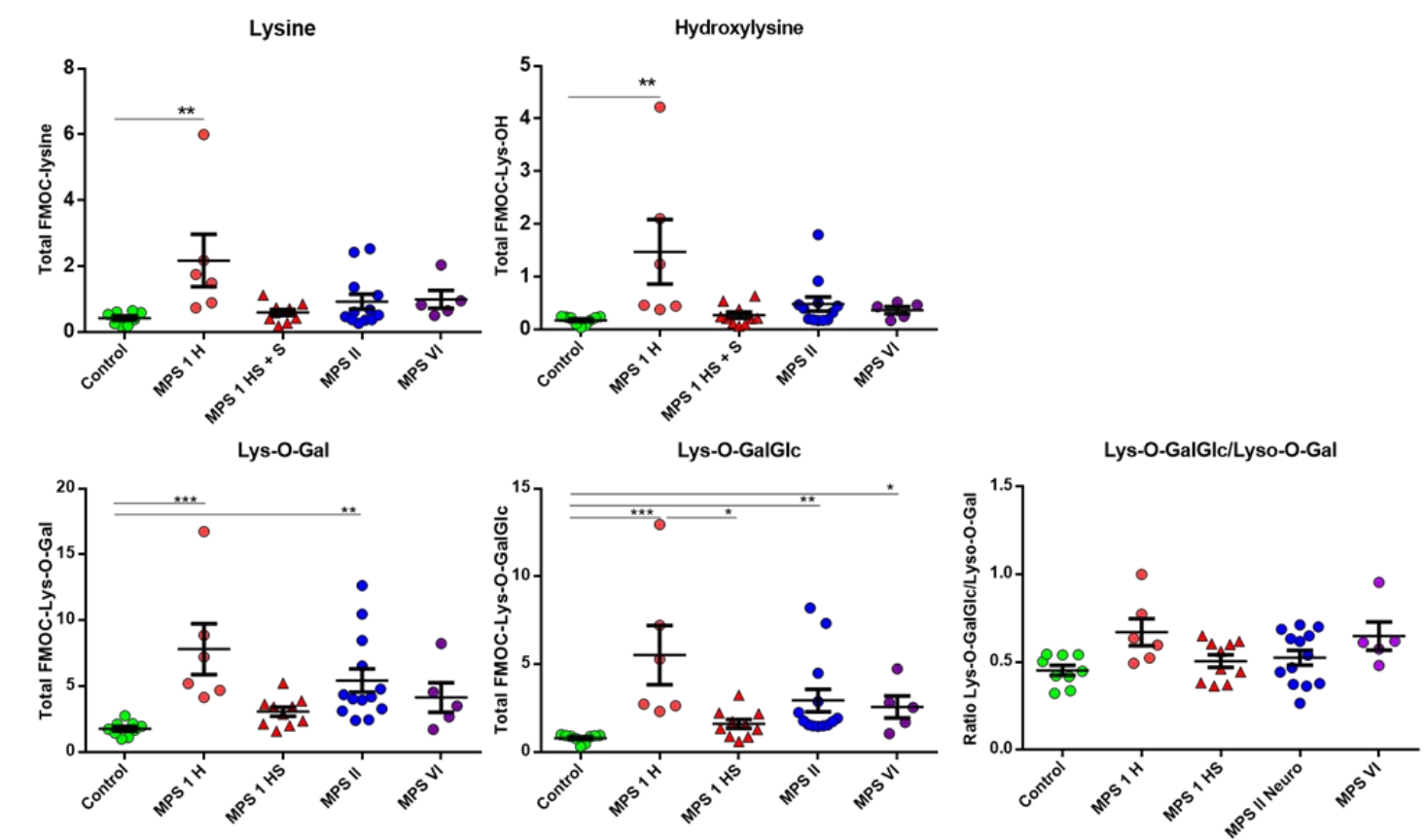

Figure 1. 


\section{Relationship of free urinary glycosylated hydroxylysine with age and urinary glycosaminoglycans}

Further analysis was performed to determine the effect of age on the concentration of urinary glycosylated hydroxylysine. The samples analysed were from MPS I, II and IVA patients and, unlike the pilot study, all of the samples had been collected prior to treatment. Figure 2A shows the effect of age on the concentration of urinary Lys-O-Gal and Lys-O-GalGlc for control $(n=41)$ and pre-treatment MPS patients $(n=37)$. Concentrations of free glycosylated hydroxylysine are highest in children $<2$ years of age after which this falls considerably and further drops until approximately 15 - 20 years of age where the levels are the lowest and remain low. This pattern is similar to that observed for urinary GAGs (de Jong et al 1989; Dave et al 2015). Most urinary markers normalised to creatinine decrease with age, reflecting a change in relative muscle mass to body mass ratio with age.

Age-related control ranges, based on the lower 5\% and upper 95\% percentile values, for LysO-Gal were 2.99-36.24 mmol/creatinine for 0 - 2 years ( $\mathrm{n}=12$ samples), 0.74-23.72 $\mathrm{mmol} /$ creatinine for 2 - 15 years $(\mathrm{n}=13$ samples) and $<5.50 \mathrm{mmol} / \mathrm{creatinine} \mathrm{for} \mathrm{adults}$ above 15 years of age ( $\mathrm{n}=16$ samples). For Lys-O-GalGlc these ranges were 1.40-27.96 $\mathrm{mmol} /$ creatinine for 0 - 2 years, 0.42-16.98 mmol/creatinine for $2-15$ years and $<4.33$ mmol/creatinine for adults above 15 years. The ratio of Lys-O-Gal/Lys-O-GalGlc (figure 2A (iii)) did not show any differences with respect to age until adulthood (> 20 years of age) when it was found to increase; 0.29-0.94 for $<20 y$ rs and 0.76-1.28 for controls $>20 y$ rs. Treatment-naive MPS samples are also shown and clearly indicate higher levels irrespective of the age of the MPS patient. 
Concentrations of glycosylated hydroxylysine in pre-treatment samples were compared to total urinary GAG levels to see if any correlation existed. Figure 2B shows that total urinary GAG levels correlate with the levels of both glycosylated hydroxylysine species $\left(r^{2}>0.7\right.$, $\mathrm{p}<0.001$ ) in pre-treatment samples from MPS patients. When looking more specifically at Keratan sulphate levels as measured by LC-MS/MS in MPS IVA patients there was a strong correlation with Lys-O-Gal although the relationship with Lys-O-GalGlc was not as well defined. We were not able to compare the concentration of the glycosylated hydroxylysines with individual Heparan Sulphate and Dermatan Sulphate levels as this information was not available. 
Figure 2
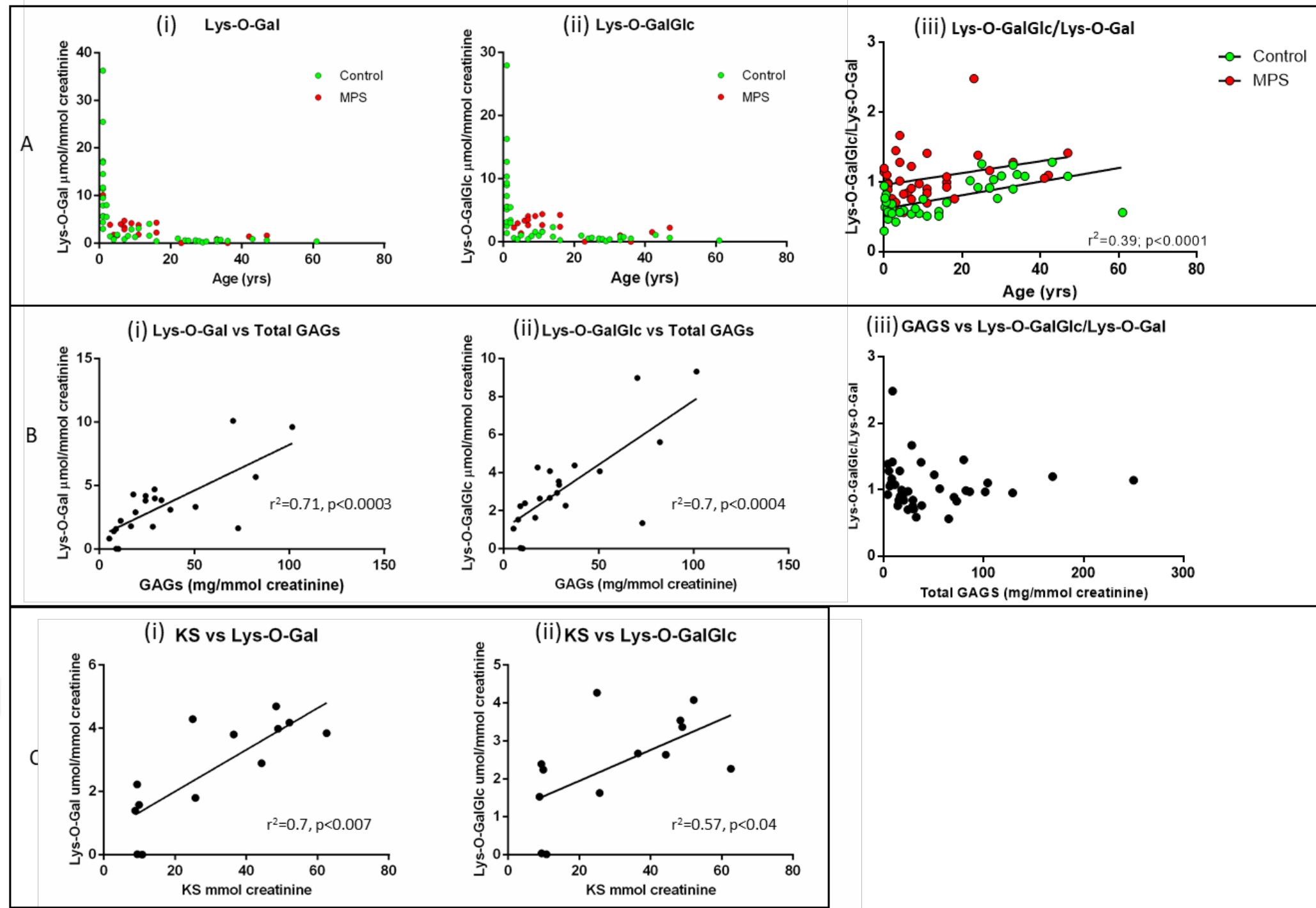

This article is protected by copyright. All rights reserved. 


\section{Confirmation of elevated urinary free glycosylated hydroxylysine in MPS using a separate validation cohort}

Samples used to assess the age relevance of the glycosylated hydroxylysines were analysed and compared to controls to confirm the observations from the pilot analysis (figure 3). Samples were grouped according to age. The MPS I group which consisted of mostly Hurler samples did not contain enough Hurler-Scheie samples to be able to further divide into the disease based sub-groups Hurler and Hurler-Scheie. Therefore the MPS I samples are collectively grouped as 'MPS I'. Both MPS I and II show highly variable levels of both glycosylated hydroxylysine species within groups for both age ranges in MPS II $(<2$ years and 2-15 years) and <2yrs for MPS I. . Whilst the levels of glycosylated hydroxylysines in MPS I and MPS II patients were elevated this was not statistically significant due to small sample numbers and disease heterogeneity. The two HS samples in the MPS I group had elevated levels and one Hurler in the $<2$ yr group had levels in the control range. Samples that had high levels in the < 2yrs MPS II group were predicted to have severe disease by a previous sibling or due to having a severe mutation (supplementary data 1). The MPS IVA group showed variation within the 2-15 years age range but was significantly increased at $>15$ years.. The Lys-O-GalGlc/Lys-O-Gal ratio (figure 3 (iii)) of the paediatric control group corroborated the findings of the pilot study, with a ratio of approximately 0.5 observed. This ratio increases to approximately 1 in the adult control group (figure 3 (iii)). The age-matched MPS groups all showed significant increases in Lys-O-GalGlc/Lys-O-Gal ratio, relative to the controls, even for samples that did not have elevated glycosylated hydroxylysine levels. Whilst statistically there is a significant difference between the adult MPS IV group and the 
control group, there was a considerable overlap with the upper control range of the adult controls. 
Figure 3.
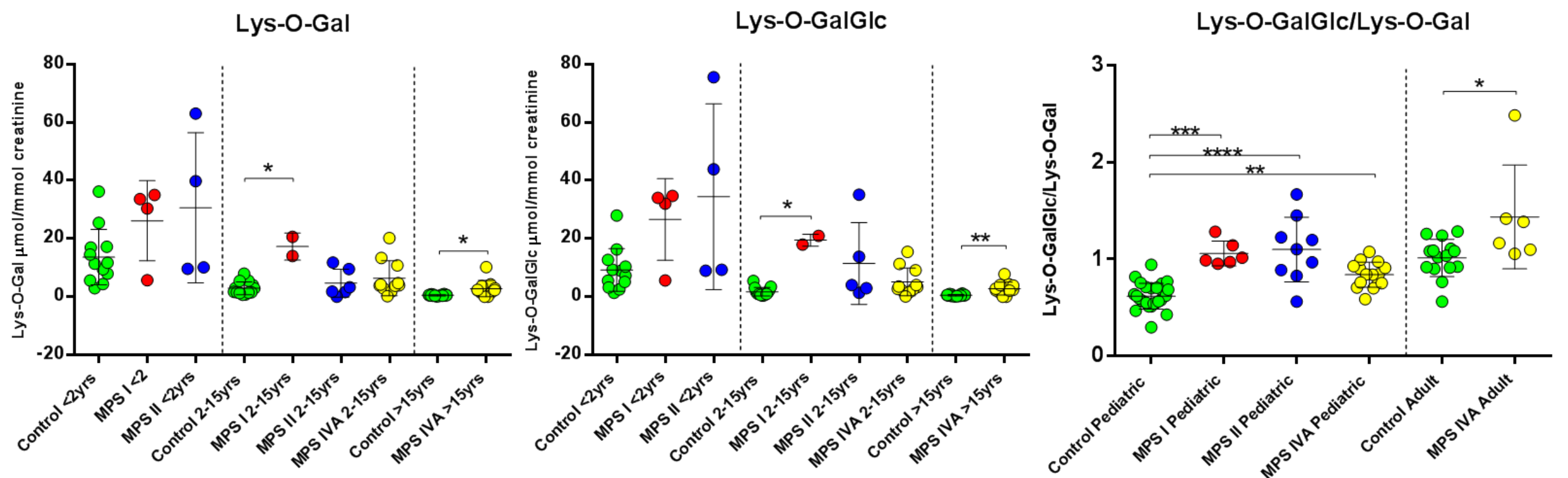


\section{Free urinary glycosylated hydroxylysine changes in other paediatric metabolic disorders}

In order to determine whether elevated levels of urinary glycosylated hydroxylysines were specific to the MPS disorders we assessed samples used previously in figure 3 from MPS I and II patients (age ranges $<2$ yrs and 2-15 years old) and an MPS III patient, which predominantly has a neurological phenotype (age range 2-15 years old) alongside cohorts of other metabolic disease samples (figure 4). The metabolic disease samples were from patients with pyridoxine-dependent epilepsy (PDE) due to mutations in ALDH7A1 (a non-lysosomal storage disorder) and lysosomal storage disorders including Fabry disease, Niemann Pick C disease and Ceroid Lipofuscinosis 2.

Figure 4 shows that MPS I and II were the only disorders with significantly elevated glycosylated hydroxylysine levels for both age groups relative to control samples. One PDE patient had levels of Lys-O-GalGlc above the upper control range. The ratio of the glycosylated hydroxylysines was also significantly increased in the MPS I and II patients compared to controls, confirming previous findings. The levels in the other metabolic disorders investigated were similar to the control range apart from 3 PDE patients just above the upper control range and an NPC patient who had a markedly elevated level within the MPS range. Hence elevation of these markers is not specific to LSDs but possibly occurs as a result of a downstream effect which is more related to disease pathology. 

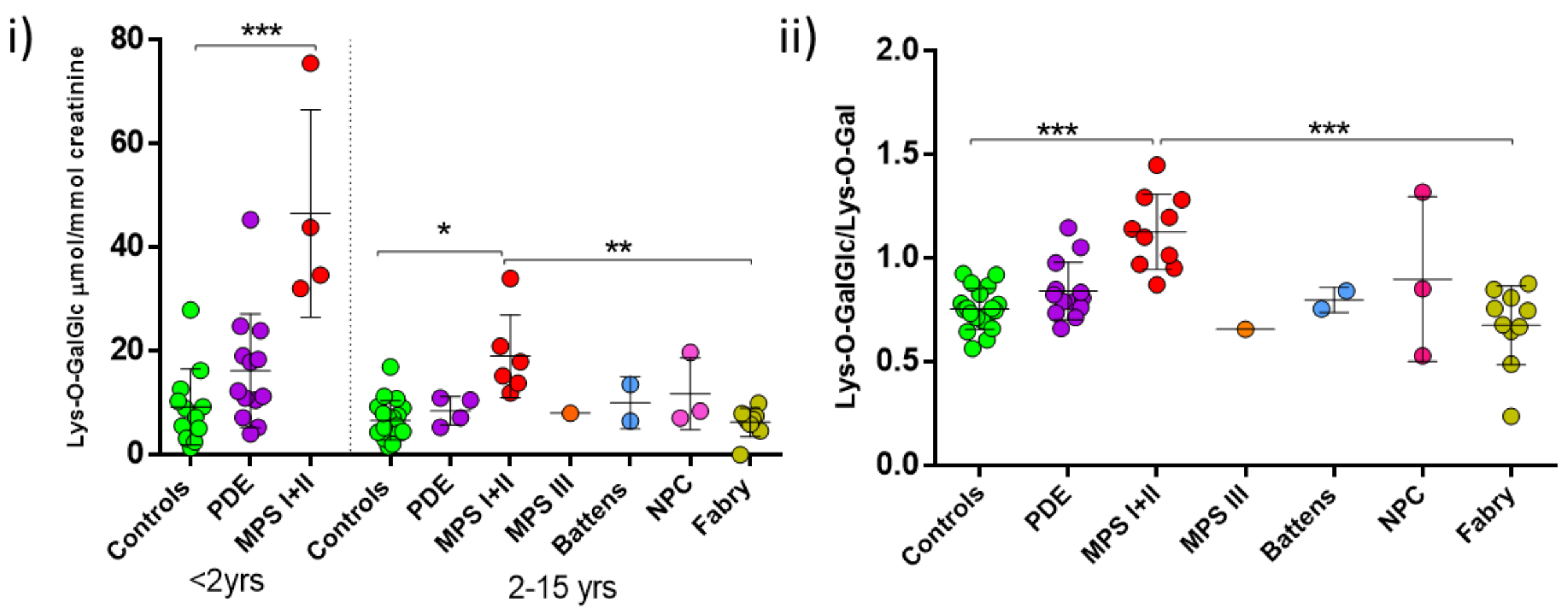


\section{Discussion}

A reduction of GAG levels is often the only way of determining treatment efficacy in MPS patients as this is the result of the primary defect in most MPS disorders (Lehman et al 2011; Lawrence et al 2014). However, it is becoming more apparent in rare diseases such as the MPS disorders that GAGs are only an instigator of a much more complex cascading pathology (Clarke 2011) and that use of GAG levels do not always correlate with disease severity (Valstar et al 2010). Further understanding of how accumulated GAGs cause the resulting pathology is needed. One key early pathological feature is the disruption of the ECM (Batzios et al 2012; Heppner et al 2015; Heywood et al 2015). The resulting consequence of this disruption needs to be further understood.

The original method for analysing Lys-O-Gal and Lys-O-GalGlc, used fractionated lyophilised samples and a single column colorimetric absorbance assay (Pinnell et al 1971). This procedure required much larger volumes of sample (1-5 ml urine) compared to the mass spectrometric method used in this analysis $(60 \mu \mathrm{l})$ and was a much longer (4 hrs) and labour intensive method. Whilst these early methods achieved a CV of 0.5-3\% (Miller and Piez, 1966) the LC-MS/MS method described here is a much quicker and more sensitive, specific and accurate method with the ability to include internal standards. Our observations of the ratio of Lys-O-Gal and Lys-O-GalGlc in human urine are comparable to that described by Krane et al in 1977 (Krane et al 1977). 
The pilot study analysis quantitating all four collagen lysines demonstrated that whilst free lysine and Lys-OH were elevated in MPS I Hurler, it was the glycosylated hydroxylysine species that was affected in most of the MPS disorders and thus more informative. Urinary lysine and Lys-OH are not derived exclusively from collagen which may explain their lack of specificity. Therefore the main focus of this research concentrated on the glycosylated hydroxylysines. Subsequent analysis of a cohort of treatment naïve MPS patients was able to confirm altered collagen turnover in MPS. There was a more profound change in the ratio of Lys-O-GalGlc/Lys-O-Gal in this treatment naïve group that was not observed in the pilot cohort. It is possible that this could be due to the pilot cohort consisting of a mix of pre- and post-treatment samples. If this is the case then this marker could potentially be treatment responsive. However, it will only be possible to confirm this by studying longitudinal samples from patients undergoing treatment. This will be the focus of future analysis.

Collagen is post-translationally modified prior to secretion and its turnover is a tightly regulated process. Overproduction and/or reduced degradation results in fibrosis and under production and/or increased degradation contributes to aging and tissue damage (Bradley et al 1975). The exact function of the glycosylated collagen is not fully understood but thought to be related to the crosslinking of collagen fibrils. The breakdown of collagen happens either extracellularly and is mediated by matrix metalloproteases or intracellularly and primarily via endo-lysosomal degradation (Everts et al 1996) which may explain its association with urinary GAG levels. Glycosylated collagen however seems to have a more specific mechanism of intracellular degradation mediated by the uPARAP/Endo180 receptor (Jurgensen et al 2011). Disturbance of the extracellular matrix breakdown in MPS disorders 
has been described by the observation of altered levels and tissue distribution of cathepsins and matrix metalloproteases in MPS disease models and patients (Batzios et al 2012;

Gonzalez et al 2018). This study indicates that the intracellular breakdown of collagen is also affected, but not necessarily as a result of lysosome dysfunction; elevated levels of glycosylated hydroxylysine suggest an increase in lysosomal collagen breakdown. Gonzalez et al (Gonzalez et al 2018) recently described the increase and altered cellular distribution of cathepsin B, a known protease of collagen IV (Shoji et al 2014) in the cellular endolysosomal system of MPS I mice. This could account for the increase of intracellular collagen breakdown and the greater specificity of this marker for MPS disorders (figure 4). Mislocalised lysosomal cathepsins in the cytosol have also been reported in NPC patient fibroblasts, which may explain the NPC patient who demonstrated an elevated ratio (Chung et al 2016).

Krane et al 1977 determined that bone and skin have specific Lys-O-GalGlc to Lys-O-Gal ratios of 0.5 and 2, respectively. The ratio of the Lys-O-GalGlc to Lys-O-Gal in our study confirms these findings, with normal paediatric subjects primarily secreting glycosylated hydroxylysine originating from bone due to growth. This changes with age, with an increasing ratio indicating contributions from non-bone tissue sources. In the MPS patients, if elevated levels of glycosylated hydroxylysines were arising due to bone degradation, it would be expected that the total levels would increase, and the ratio remain unchanged or decrease due to a greater contribution of bone derived Lys-O-Gal as would be expected in diseases of bone degradation/loss such as osteoporosis, juvenile arthritis or Pagets disease as shown by (Krane et al 1977). However, bone pathology in MPS differs to that which occurs in other 
rheumatological diseases such as inflammatory arthritis, with degradation observed more in the connective tissue such as ligaments, tendons and joint capsules of MPS patients (Morishita and Petty 2011). It is possible that the increased ratio of Lys-O-GalGlc/Lys-O-Gal in MPS patients suggests contribution of glycosylated hyroxylysines not from the bone but the bone associated tissues such as cartilage and connective tissue for which disruption is a key cause of the pathological skeletal phenotype observed in MPS I, II, IV and VI patients (Kelly et al 2013; Wang et al 2014; Yano et al 2014). The observation that the MPS III patient tested (figure 4) showed glycosylated hyroxylysines levels within control range also indicates that these markers may well be associated with the skeletal pathology as MPS III patients have primarily a neurological disease phenotype.

One limitation of this study is the limited availability of MPS samples from patients who are treatment naïve as typically newly diagnosed patients start treatment shortly after diagnosis. This has made determining the importance of these markers difficult. The well-known issue of clinical heterogeneity of disease pathology within the MPS disorders (Stapleton et al 2018) also confounds this further, as not all patients will present with the same pathological features and to the same extent. This limits our ability to correlate altered concentrations of urinary glycosylated hydroxylysines with a specific pathology. Further assessment of glycosylated hydroxylysine as a potential biomarker for the monitoring of response to treatment and clinical outcome will be required in the future. Further work is also required to understand the underlying pathological association of and hence implication of increased intracellular breakdown of collagen in MPS disorders. Blood and CSF could also potentially be a resource 
of glycosylated hydroxylysines that could indicate treatment response on more specific organs such as the brain. This could be useful for monitoring new intrathecal treatments in MPS (Giugliani et al 2018).

\section{Acknowledgments}

All research at Great Ormond Street Hospital NHS Foundation Trust and UCL Great Ormond Street Institute of Child Health is made possible by the NIHR Great Ormond Street Hospital Biomedical Research Centre. The views expressed are those of the author(s) and not necessarily those of the NHS, the NIHR or the Department of Health. This work was also partly supported by an IIR award from Shire Pharmaceuticals UK. Lys-O-GalGlc and LysO-Gal standards were a kind gift from Professor Ruggero Tenni (University of Pavia, Italy).

\section{Supplementary Data}

Supplementary Data 1: Sample information and data

Supplementary Data 2:

Supplementary Table S1: Mass Spectrometry Parameters

Supplementary Table S2: Analyte transitions

Supplementary Table S3: Control ranges for total urinary GAGs and keratan sulphate used by Great Ormond Street Clinical Pathology laboratories

Figure S1: Example chromatograms of lysine, lys-OH, lys-O-Gal, Lys-O-GalGlc

Figure S2: Standard curves for lysine, lys-OH, lys-O-Gal, Lys-O-GalGlc

Table S4: Interbatch CV data for a pooled urine sample injected 13 times

Table S5: Intrabatch CV data for 4 urine samples (A-D) injected on 5 different days 


\section{References}

Banushi B, Forneris F, Straatman-Iwanowska A, et al (2016) Regulation of post-Golgi LH3 trafficking is essential for collagen homeostasis. Nat Commun 7: 12111.

Batzios SP, Zafeiriou DI, Vargiami E, Karakiulakis G, Papakonstantinou E (2012) Differential expression of matrix metalloproteinases in the serum of patients with mucopolysaccharidoses. JIMD Rep 3: 59-66.

Bradley K, McConnell-Breul S, Crystal RG (1975) Collagen in the human lung. Quantitation of rates of synthesis and partial characterization of composition. The Journal of clinical investigation 55: 543-550.

Chung C, Puthanveetil P, Ory DS, Lieberman AP (2016) Genetic and pharmacological evidence implicates cathepsins in Niemann-Pick C cerebellar degeneration. Human molecular genetics 25: 1434-1446.

Clarke LA (2011) Pathogenesis of skeletal and connective tissue involvement in the mucopolysaccharidoses: glycosaminoglycan storage is merely the instigator. Rheumatology 50 Suppl 5: v13-18.

Dave MB, Chawla PK, Dherai AJ, Ashavaid TF (2015) Urinary Glycosaminoglycan Estimation as a Routine Clinical Service. Indian J Clin Biochem 30: 293-297.

de Jong JG, Wevers RA, Laarakkers C, Poorthuis BJ (1989) Dimethylmethylene blue-based spectrophotometry of glycosaminoglycans in untreated urine: a rapid screening procedure for mucopolysaccharidoses. Clinical chemistry 35: 1472-1477.

Everts V, van der Zee E, Creemers L, Beertsen W (1996) Phagocytosis and intracellular digestion of collagen, its role in turnover and remodelling. Histochem J 28: 229-245.

Giugliani R, Vairo F, Kubaski F, et al (2018) Neurological manifestations of lysosomal disorders and emerging therapies targeting the CNS. The Lancet Child \& adolescent health 2: 56-68.

Gonzalez EA, Martins GR, Tavares AMV, et al (2018) Cathepsin B inhibition attenuates cardiovascular pathology in mucopolysaccharidosis I mice. Life Sci 196: 102-109.

Heppner JM, Zaucke F, Clarke LA (2015) Extracellular matrix disruption is an early event in the pathogenesis of skeletal disease in mucopolysaccharidosis I. Molecular genetics and metabolism 114: 146-155.

Heywood WE, Camuzeaux S, Doykov I, et al (2015) Proteomic Discovery and Development of a Multiplexed Targeted MRM-LC-MS/MS Assay for Urine Biomarkers of Extracellular Matrix Disruption in Mucopolysaccharidoses I, II, and VI. Analytical chemistry.

Jurgensen HJ, Madsen DH, Ingvarsen S, et al (2011) A novel functional role of collagen glycosylation: interaction with the endocytic collagen receptor uparap/ENDO180. The Journal of biological chemistry 286: 32736-32748.

Kelly AS, Metzig AM, Steinberger J, Braunlin EA (2013) Endothelial function in children and adolescents with mucopolysaccharidosis. J Inherit Metab Dis 36: 221-225.

Krane SM, Kantrowitz FG, Byrne M, Pinnell SR, Singer FR (1977) Urinary excretion of hydroxylysine and its glycosides as an index of collagen degradation. The Journal of clinical investigation 59: 819-827.

Lawrence R, Brown JR, Lorey F, Dickson PI, Crawford BE, Esko JD (2014) Glycan-based biomarkers for mucopolysaccharidoses. Molecular genetics and metabolism 111: 73-83.

Lehman TJ, Miller N, Norquist B, Underhill L, Keutzer J (2011) Diagnosis of the mucopolysaccharidoses. Rheumatology 50 Suppl 5: v41-48.

Mills PB, Struys E, Jakobs C, et al (2006) Mutations in antiquitin in individuals with pyridoxine-dependent seizures. NatMed 12: 307-309.

Morishita K, Petty RE (2011) Musculoskeletal manifestations of mucopolysaccharidoses. Rheumatology 50 Suppl 5: v19-25.

Oussoren E, Brands MM, Ruijter GJ, der Ploeg AT, Reuser AJ (2011) Bone, joint and tooth development in mucopolysaccharidoses: relevance to therapeutic options. Biochimica et biophysica acta 1812: $1542-$ 1556.

Pinnell SR, Fox R, Krane SM (1971) Human collagens: differences in glycosylated hydroxylysines in skin and bone. Biochimica et biophysica acta 229: 119-122. 
Shoji A, Kabeya M, Ishida Y, Yanagida A, Shibusawa Y, Sugawara M (2014) Evaluation of cathepsin B activity for degrading collagen IV using a surface plasmon resonance method and circular dichroism spectroscopy. J Pharm Biomed Anal 95: 47-53.

Spiro RG (1969) Characterization and quantitative determination of the hydroxylysine-linked carbohydrate units of several collagens. The Journal of biological chemistry 244: 602-612.

Stapleton M, Arunkumar N, Kubaski F, Mason RW, Tadao O, Tomatsu S (2018) Clinical presentation and diagnosis of mucopolysaccharidoses. Molecular genetics and metabolism 125: 4-17.

Valstar MJ, Neijs S, Bruggenwirth HT, et al (2010) Mucopolysaccharidosis type IIIA: clinical spectrum and genotype-phenotype correlations. Annals of neurology 68: 876-887.

Wang RY, Braunlin EA, Rudser KD, et al (2014) Carotid intima-media thickness is increased in patients with treated mucopolysaccharidosis types I and II, and correlates with arterial stiffness. Molecular genetics and metabolism 111: 128-132.

Yano S, Moseley K, Wong L, Castelnovi C, Azen C, Pavlova Z (2014) Glycosaminoglycan metabolism defects and atherosclerosis: frequent association of endothelial dysfunction in patients with Mucopolysaccharidosis. J Inherit Metab Dis 37: 255-261.

Figure 1. MPS Test cohort analysis. A. A schematic of the enzymatic process of lysine glycosylation. Lysine is hydroxylated by lysyl hydroylases LH1-3. Hydroxylated lysine is glycosylated by procollagen galactosyltransferase 1 \& 2 and LH3. B. LC-MS/MS analysis of the modified lysines, depicted in Figure 1A, in MPS urine samples. MPS I is subdivided into Hurler (MPS I), moderate /mild Hurler -Scheie (MPS I HS+S). Significance is determined by non-parametric Kruskal Wallis ${ }^{*} \mathrm{p}<0.05,{ }^{* *} \mathrm{p}<0.01,{ }^{* * *} \mathrm{p}<0.001$

\section{Figure 2. Relationship of free urinary glycosylated hydroxylysine with age and}

glycosaminoglycans. A. The effect of age on the total levels of free urinary glycosylated hydroxylysine and on the ratio of Lys-O-Gal/Lys-O-GalGlc in control $(n=41)$ and combined group of MPS I, II and IVA ( $\mathrm{n}=37)$ samples. Levels are highest in children under 2 years and lowest in adults $>20$ years. The ratio of Lys-O-GalGlc/Lys-O-Gal does not significantly 
change until adulthood ( $>20$ years) B. Pearson correlation analysis shows there is a strong and significant association between total urinary GAGs with glycosylated hydroxylysine in untreated MPS patients but there is no association of the ratio of the glycosides with GAG levels. C. Keratan sulphate levels correlate more strongly for Lys-O-Gal in untreated MPS IVA patients.

\section{Figure 3. Confirmation of altered modified hydroxylysine in pre-treatment MPS}

patients. Additional pre-treatment patient samples were subdivided into age groups specified in the age correlation analysis. i) Lys-O-Gal shows less difference in MPS patients relative to controls with only increased levels observed in MPS I at 2-15 years and MPS IVA in >15 years. ii) Urinary Lys-O-GalGlc levels are variable in the $<2$ years age group for MPS I and II. Significantly higher levels are also observed for MPS I at 2-15 years and for MPS IVA at $>15$ years. iii) The ratio of Lys-O-GalGlc to Lys-O-Gal is significantly different in the paediatric MPS groups and also in adult MPS IVA groups relative to the control cohort. Significance determined by non-parametric testing * $\mathrm{p}<0.05{ }^{* *} \mathrm{p}<0.01,{ }^{* * *} \mathrm{p}>0.001$, $* * * * \mathrm{p}<0.0001$

Figure 4. Urinary glycosylated hydroxylysine in other metabolic disorders. i) Analysis of samples from individuals $<2$ years of age shows that levels of Lys-O-GalGlc are elevated in MPS patients relative to controls and patients with PDE. The MPS group still has the highest levels in the 2-15 years age group compared with other metabolic disorders. ii) Ratio of Lys- 
O-GalGlc to Lys-O-Gal shows that MPS patients demonstrate altered ratios compared to other metabolic disorders that ordinarily do not have a strong mucoskeletal disease phenotype. 